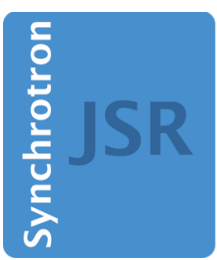

JOURNAL OF

SYNCHROTRON

RADIATION

Volume 27 (2020)

Supporting information for article:

LamNI - an instrument for X-ray scanning microscopy in laminography geometry

Mirko Holler, Michal Odstrčil, Manuel Guizar-Sicairos, Maxime Lebugle, Ulrich Frommherz, Thierry Lachat, Oliver Bunk, Joerg Raabe and Gabriel Aeppli 


\section{LamNI - an instrument for X-ray scanning microscopy in laminography geometry}

\section{Supporting Figures}

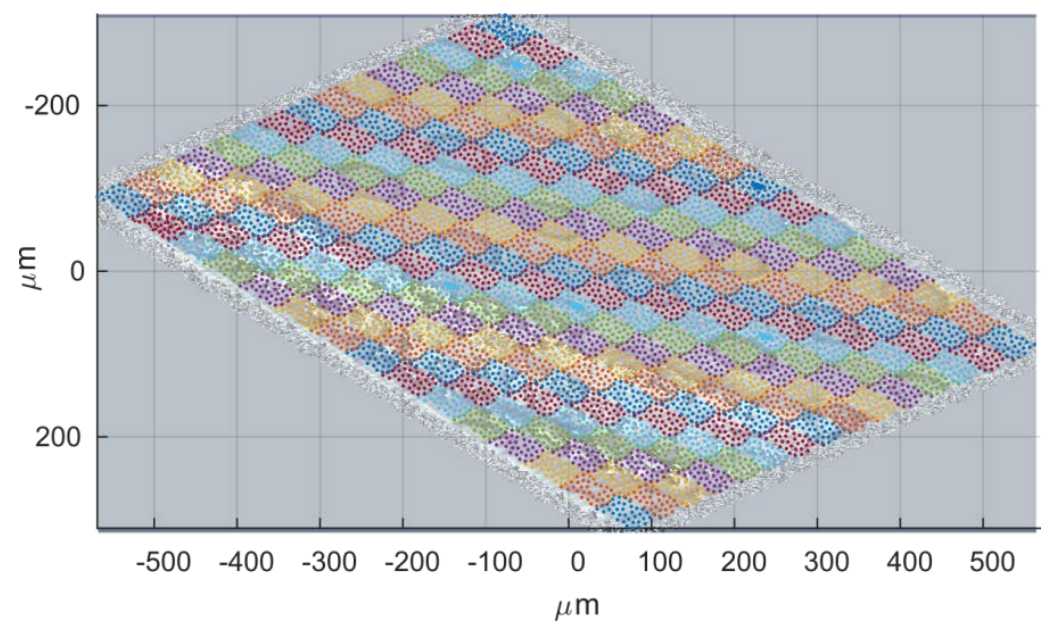

Figure S1 A demonstration of the stitching capability of the instrument. The figure shows a single projection with a square shape about $(0.5 \mathrm{~mm})^{2}$ in size in the sample plane. The data was acquired by scanning many smaller areas using the Piezo stage and stitching such scans using the coarse stages. The individual sub-scans are indicated by differently colored symbols. 